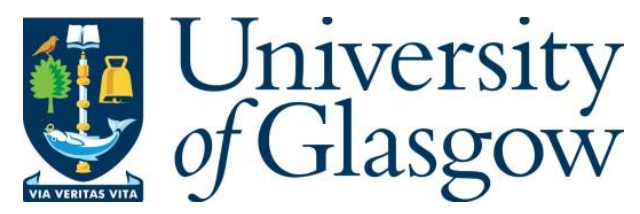

Macdonald, S. A. and Brewster, S. (2019) Gamification of a To-Do List with Emotional Reinforcement. 37th Annual ACM CHI Conference on Human Factors in Computing Systems (CHI '19 Extended Abstracts), Glasgow, UK, 04-09 May 2019. ISBN 9781450359719.

There may be differences between this version and the published version. You are advised to consult the publisher's version if you wish to cite from it.

(C) The Authors 2019. This is the author's version of the work. It is posted here for your personal use. Not for redistribution. The definitive Version of Record was published in the Proceedings of the 37th Annual ACM CHI Conference on Human Factors in Computing Systems (CHI '19 Extended Abstracts), Glasgow, UK, 04-09 May 2019. ISBN 9781450359719. http://dx.doi.org/10.1145/3290607.3313060.

\title{
http://eprints.gla.ac.uk/184005/
}

Deposited on: 15 April 2019

Enlighten - Research publications by members of the University of Glasgow http://eprints.gla.ac.uk 


\section{Gamification of a To-Do List with Emotional Reinforcement}

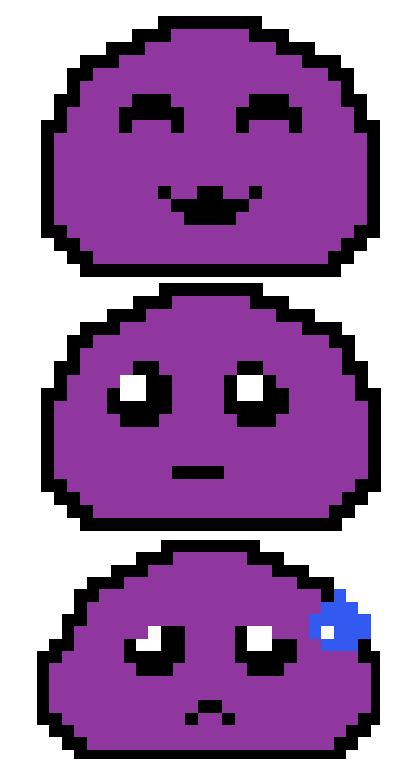

Figure 1: Single frames from Tamu's longterm mood animations, designed to deliver emotional reinforcement based on the user's actions [11]

\section{Shaun Macdonald}

Glasgow Interactive Systems Section

School of Computing Science

University of Glasgow, Scotland

s.macdonald.5@research.gla.ac.uk

mig.dcs.gla.ac.uk

\author{
Stephen Brewster \\ Glasgow Interactive Systems Section \\ School of Computing Science \\ University of Glasgow, Scotland \\ stephen.brewster@glasgow.ac.uk \\ mig.dcs.gla.ac.uk
}

\begin{abstract}
Gamification can change how and why people interact with software. A common approach is to use quantitative feedback to give users a feeling of progress or achievement. There are, however, other ways to provide users with motivation or meaning during normal computer interactions, such as using emotional reinforcement. This could provide a powerful new tool to allow the positive effects of gamification to reach wider contexts. This paper investigates the design and evaluation of a mobile to-do list application, 'Tamu To-Do', which utilises gamified emotional reinforcement, as seen in Figure 1. A week-long field study $(\mathrm{N}=9)$ recorded user activity and impressions with the application. The results supported emotional reinforcement's potential as a gamification strategy to improve user motivation and engagement.
\end{abstract}

\section{CCS CONCEPTS}

- Human-centered computing $\rightarrow$ Field studies; User centered design; • Software and its engineering $\rightarrow$ Interactive games;

\section{CHI'19 Extended Abstracts, May 4-9, 2019, Glasgow, Scotland Uk}

2019. This is the author's version of the work. It is posted here for your personal use. Not for redistribution. The definitive Version of Record was published in Proceedings of CHI Conference on Human Factors in Computing Systems Extended Abstracts (CHI'19 Extended Abstracts), https://doi.org/10.1145/3290607.3313060. 


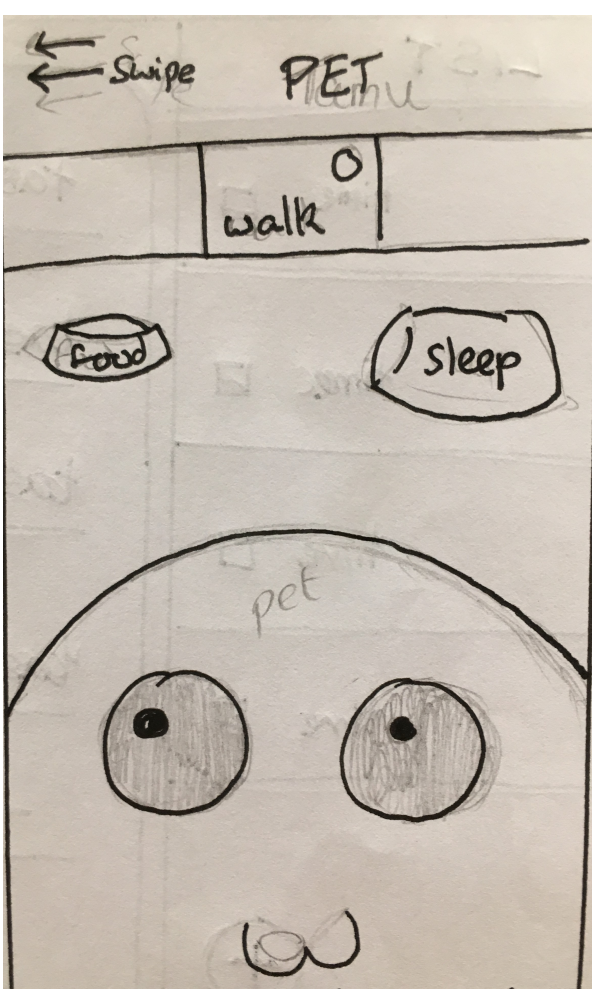

Figure 2: One of many early sketch prototypes during Iteration 1 of application development.

\section{KEYWORDS}

Gamification; Emotional Reinforcement; Motivation; Mobile $\mathrm{HCl}$

\section{ACM Reference Format:}

Shaun Macdonald and Stephen Brewster. 2019. Gamification of a To-Do List with Emotional Reinforcement. In Proceedings of CHI Conference on Human Factors in Computing Systems Extended Abstracts (CHI'19 Extended Abstracts). ACM, New York, NY, USA, 6 pages. https://doi.org/10.1145/3290607.3313060

\section{INTRODUCTION}

Personal organisation is a major concern for many in modern society and keeping track of responsibilities and tasks is challenging. Gamification can help users stay motivated through a continuous stream of new goals and focus on specific tasks [12]. The majority of gamified applications use quantitative metrics to convey progress and motivate users towards milestones or goals. However, there may be other ways to use gamification to improve user engagement.

Emotional reinforcement, the use of emotional feedback to alter future behaviour, has been shown across other disciplines to have potential [10] [9]. However, it has been poorly explored for gamification, with only scarce and niche products and a dearth of specific research and experimental evaluation This paper tackles this space by contributing a field study evaluation of a gamified productivity application which utilises emotional reinforcement. The productivity application chosen was a to-do list due to the wide range of contexts in which it can be used. It is also a known and understood tool for the majority of users. Our hypothesis is that gamification of a productivity application using emotional reinforcement will improve user motivation and engagement.

\section{RELATED WORK}

Gamification is defined as "the use of game design elements in non-game contexts" [3] and is often used to increase user engagement. Common elements from games are point-scoring, levelling systems, leaderboards, achievements and other measures which may symbolise the user's progress with a task by relating it to making progress in a game.

The research landscape around gamification is still emerging as many possible mechanisms, such as emotional reinforcement, are mostly unexplored. The majority of the studies that assess the effectiveness of gamification have been shown to increase average motivation, accessibility, focus and engagement with applications [2, 4, 6-8]. All of this research studied gamified applications that used quantitative progression towards in-game goals to reward user engagement with the core application For example, in Fitz-Walter et al.'s Driven to Drive [4], user progress as they learn to drive is visualised as progress along an in-game road trip. Other studies in this area are more exploratory, such as 


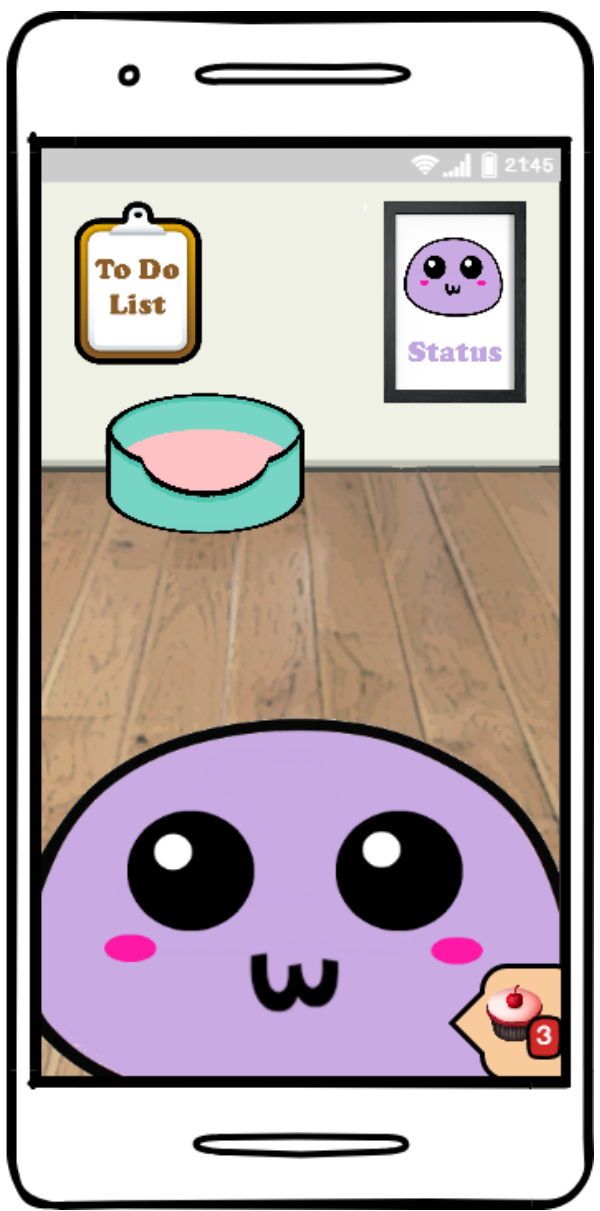

Figure 3: Final prototype iteration before implementation of the design, after 3 formative evaluations alongside users helped to shape the design.
Gonczarowski et al.'s Incentives and Gamification [5], which outlines examples of incentivising users with gamification and its implications, but without presenting evidence of their efficacy.

Stinson et al. [13] made use of role-playing game elements in their application Pain Squad to help children with cancer record their pain symptoms. This application was shown to be successful in increasing patient engagement with pain tracking and did feature emotional reinforcement elements. After completing a task, a video would play to the child featuring an actor in the game universe thanking them for their efforts and encouraging them. This application of emotional reinforcement is narrow in its context and target user base, but showed potential for the use of emotional reinforcement as a gamification reward.

There are examples of successful commercial applications that use gamification to promote productivity, such as Duolingo (www.duolingo.com), Habitica (www.habitica.com), Super Better (www.superbetter.com), To-Doist (todoist.com/), Epic Win (www.rexbox.co.uk/epicwin/) and Forest (www.forestapp.cc/en/). All these applications use numerical measures to symbolise player progress and do not feature emotional reinforcement elements. Duolingo states they have over 120 million users worldwide using this qualitative strategy [1]. Positive emotional reinforcement has proven positive effects [10] [9], but is little explored in gamification. While receiving progression based rewards such as badges, experience and levels can be seen as positive reinforcement for the user, these rewards are not emotional in nature. Giving the user purely emotional feedback as reinforcement may have potential.

\section{APPLICATION DEVELOPMENT}

An application was created in order to test the project hypothesis. The basic requirement was a simple to-do list combined with a virtual pet that was designed to deliver emotional reinforcement based on usage of the list. The design for the application was created over the course of 4 iterations.

Each iteration following the first featured a design stage where information from prior formative evaluation was used to narrow down and develop previous wireframed ideas. This was followed by a formative evaluation stage where users were asked to select which design they preferred and why in a followup discussion. Users were recruited from social spaces on the University of Glasgow campus for each iteration, with 25 participants during Iteration 1 (Figure 2), 16 participants in Iteration 2 and 11 participants for Iteration 3. After these iterations a final design, Iteration 4 (Figure 3), was used to inform the implementation of a functional prototype application (Figure 4) for iOS. This application was subject to user-acceptance testing $(\mathrm{N}=5)$ to identify potential navigation or readability issues, as well as check for bugs. Following some small technical fixes, the application was ready to use as for a field study to give longer term insights.

The final design features an animated virtual pet, named 'Tamu', living inside a virtual room. Within the room is a clipboard labelled 'To Do' which opens a to-do list allowing users to add, check off and delete their tasks. When tasks are added or completed the pet responds with positive emotional 


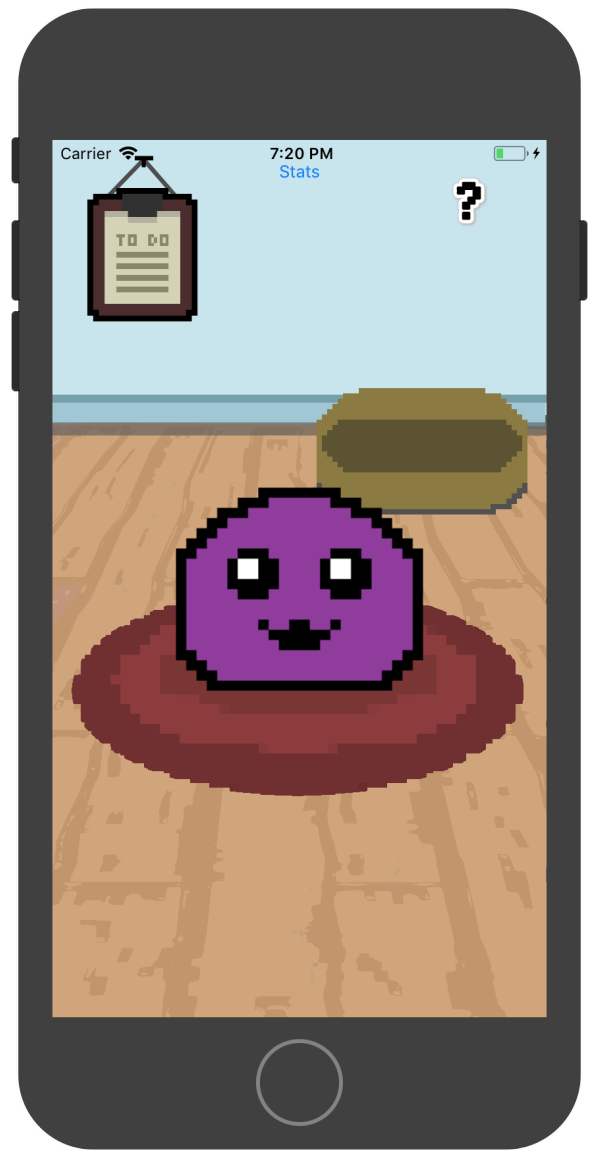

Figure 4: Tamu To-Do's home screen, showing a positive emotional display to the user, running on the XCode iOS simulator. displays. Tamu constantly displays a facial expression (Figure 1) that is either 'very happy', 'happy', 'sad' or 'very sad', based on a hidden 100-point scale. This scale gradually ticks down over time and is topped up when the user adds or completes tasks on the to-do list, with task completion being worth 20 points and adding a task worth 6 points. The point values calibrated after pilot testing, with an aim to make the system capable of delivering negative reinforcement without being too punishing and discouraging players.

Being above 75 on the scale causes the pet to be 'very happy', above 50 for 'happy', above 25 for 'sad' and below 25 for 'very sad'. To maintain a consistently positive emotional response from Tamu, the user must therefore consistently add and complete their tasks. When users add or complete tasks on the to-do list, Tamu displays short positive animations (Figure 5). If Tamu is 'happy' or 'very happy' the user can interact with the pet by tapping it, triggering a short and positive animation, or tapping the bed in its room, which will cause it to take a nap. These interactions were added to make Tamu feel more responsive and help the user form an emotional attachment to it.

\section{FIELD STUDY}

The application was evaluated in a 7-day field study $(\mathrm{N}=9)$. The primary goal was to identify if Tamu had an effect on the users behaviour and their motivation to complete tasks. Participants were recruited via advertisements on social media and from the University of Glasgow student community. $67 \%$ of participants were between $21-29$, and $11 \%$ each were $18-20,50-29$ or 60 + respectively. $44 \%$ were female and $55 \%$ were male. $78 \%$ used some form of to-do list in their daily lives.

A field study allowed for observation of the app in everyday life, with users asked to use the app to organise their life tasks for a week and encouraged to use it at least once a day but were otherwise unguided. The application logged all user actions, both updating their to-do list and also interacting with their virtual pet (tapping it gave a short animated response). It also logged when then application displayed specific emotional responses to the user. Participants were asked to self-report their impressions of the application via an online survey. This survey, which utilised 5-point Likert scales, asked about their impressions of the application, if they felt motivated by Tamu's emotional reinforcement, and whether they felt an emotional attachment to Tamu. This survey was completed after 2 days and again after 7 days. These impressions could then be compared to usage logs to see if any trends could be identified.

\section{RESULTS AND DISCUSSION}

Quantitative and qualitative performance criteria were established to focus data analysis and demonstrate possible confirmation of the project hypothesis. User engagement was measured by how many tasks they added per day, how many they completed, how often they interacted with Tamu and 


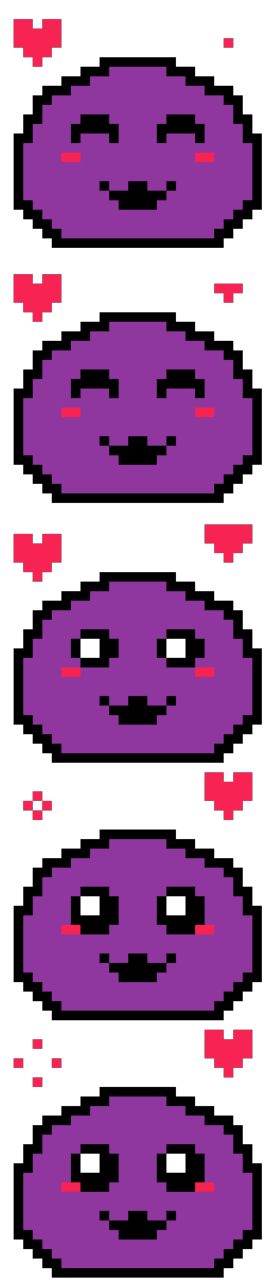

Figure 5: 5 frames used in the Tamu Love animation which plays when a user completes a task on the to-do list. This animation is made up of a total of 23 frames and takes just over 2 seconds to complete [11]. whether there was a correlation between witnessing positive or negative emotional reinforcement events and increased subsequent to-do list usage.

Complementing the usage logs from the application, users were asked to self-report on their experience with the application after 2 days and 7 days. Users were asked if they felt motivated by Tamu when they added tasks or completed tasks, if they felt motivated to be more productive when Tamu was visually unhappy and if they felt any emotional attachment to Tamu.

User engagement varied between participants, with one highly engaged user having 397 interactions with the application over the course of the week. This encompassed both the user's actions, with the to-do list and Tamu, and the emotional reinforcement events that Tamu presented to them. On average, participants had 140 interactions with the application over the week and checked the application 2.57 times a day. Users interacted with the Tamu significantly more than with the to-do list, often spending some time after checking or using their to-do list to tap Tamu and see the animation that followed.

By the end of the study, $55 \%$ of users reported feeling some emotional attachment to Tamu and $78 \%$ felt motivated by Tamu's positive reactions to completing a task. Only $56 \%$ of users witnessed a negative emotional response from Tamu, as some participants were consistently active enough to avoid it, but among those users, $67 \%$ felt it improved their motivation to be productive.

User engagement with the application reduced as the week progressed. On average, during the first two days, participants had 52 interactions with the application, on the last two days, it was 14 Given that engagement with the application's core concept was high, based on the self-reports, users were surveyed on why they used the application less. $78 \%$ felt more functionality from the to-do list was required for them to use it in future, but opinions on Tamu and the motivational effects of the emotional reinforcement were positive.

This initial study had a number of limitations that future work will address. A similar application but with more robust to-do list functionality will be tested over a longer duration, so that limitations in the to-do list functionality do not restrict usage. This will allow user perception to be based on the gamified elements rather than any shortcomings in the host application. A control group that uses the host to-do list application without any gamified elements would help us to assess how the productivity of users was affected by emotional reinforcement.

\section{CONCLUSIONS}

Some gamification strategies have been shown to improve productivity and motivation of users, but many possibilities are still unexplored. Emotional reinforcement has been used for positive behavioural change in other fields and the work here brought this to a mainstream to-do list application for the first time. Our hypothesis was that using emotional reinforcement would yield improvement motivation and engagement from users. A field study of 9 participants over 7 days showed the majority of 
participants felt emotional attachment to the virtual pet and reported it improved their motivation to complete daily tasks. This shows a clear indication that emotional reinforcement has the potential to be an effective gamification mechanism that can help users make positive behavioural change.

\section{REFERENCES}

[1] 2016. Duolingo - Which countries study which languages and what can we learn from it? Web Page. http://making. duolingo.com/which-countries-study-which-languages-and-what-can-we-learn-from-it

[2] Gabriel Barata, Sandra Gama, Joaquim Jorge, and Daniel Gonçalves. 2013. Improving participation and learning with gamification. Proceedings of the First International Conference on Gameful Design, Research, and Applications - Gamification '13 (2013), 10-17. https://doi.org/10.1145/2583008.2583010

[3] Daniel J Dubois and Giordano Tamburrelli. 2013. Understanding Gamiiiñacation Mechanisms for Software Development 2014.pdf. (2013), 659-662. https://doi.org/10.1145/2491411.2494589

[4] Zachary Fitz-Walter, Peta Wyeth, Dian Tjondronegoro, and Bridie Scott-Parker. 2013. Driven to drive. Proceedings of the First International Conference on Gameful Design, Research, and Applications - Gamification '13 (2013), 42-49. https://doi.org/10.1145/2583008.2583014

[5] Yannai A. Gonczarowski and Gustavo F. Tondello. 2017. Incentives and gamification. XRDS: Crossroads, The ACM Magazine for Students 24, 1 (2017), 9-11. https://doi.org/10.1145/3123766

[6] Dimitar Goshevski, Joana Veljanoska, and Thanos Hatziapostolou. 2017. A Review of Gamification Platforms for Higher Education. Proceedings of the 8th Balkan Conference in Informatics on - BCl '17 (2017), 1-6. https://doi.org/10.1145 3136273.3136299

[7] J Hamari and Jonna Koivisto. 2013. Social Motivations To Use Gamification : an Empirical Study of Gamifying Exercise. Proceedings of the 21st European Conference on Information Systems. ECIS 2013 (2013), 1-12. https://doi.org/10.1016/j.chb. 2015.07.031

[8] Juho Hamari, Jonna Koivisto, and Harri Sarsa. 2014. Does gamification work? - A literature review of empirical studies on gamification. Proceedings of the Annual Hawaii International Conference on System Sciences (2014), 3025-3034. https: //doi.org/10.1109/HICSS.2014.377 arXiv:arXiv:1011.1669v3

[9] Christina Kennedy and Kristine Jolivette. 2008. The Effects of Positive Verbal Reinforcement on the Time Spent Outside the Classroom for Students With Emotional and Behavioral Disorders in a Residential Setting. Behavioural Disorders 33, 4 (2008), 211-221.

[10] Ji Lu, Catherine Huet, and Laurette Dubé. 2011. Emotional reinforcement as a protective factor for healthy eating in home settings. American Journal of Clinical Nutrition 94, 1 (2011), 254-261. https://doi.org/10.3945/ajcn.110.006361

11] Shaun Macdonald. 2018. Piskel - Shaun Macdonald Public Sprite Artwork. Webpage. https://www.piskelapp.com/user/ 4747007892652032

[12] Sebastian Deterding Steffen P. Walz. 2015. The Gameful World: Approaches, Issues, Applications. MIT Press, 1 Duchess St, Marylebone, London W1W 6AN

[13] Jennifer N. Stinson, Lindsay A. Jibb, Cynthia Nguyen, Paul C. Nathan, Anne Marie Maloney, L. Lee Dupuis, J. Ted Gerstle, Benjamin Alman, Sevan Hopyan, Caron Strahlendorf, Carol Portwine, Donna L. Johnston, and Mike Orr. 2013. Development and testing of a multidimensional iphone pain assessment application for adolescents with cancer. Journal of Medical Internet Research 15, 3 (2013). https://doi.org/10.2196/jmir.2350 